\title{
Influence of Initial Stage Adhesion to Activated Sludge on Surface Modified Carbon Fiber by Photo-oxygenation
}

\author{
Tetsurou Yamada, Makoto Sato, Youichi Kamiishi, Kiyoshi Miyashita ${ }^{\dagger}$, Hironori Kaihara ${ }^{\ddagger}$, \\ Sugio Otani ${ }^{\mathbf{5}}$, Akira Kojima ${ }^{\ddagger}$, and Takao Yoshikawa* \\ Textile Research Institute of Gunma Prefecture, 5-46-1 Aioi-cho,Kiryu-City, Gunma, 376-0011,Japan \\ ${ }^{t}$ Gunma Prefectural Industrial Technology Research Laboratory,190 Toriha-cho, Maebashi-City, \\ Gunma,371, Japan \\ ${ }^{\ddagger}$ Gunma National College of Technology,580 Toriha-cho, Maebashi-City, Gunma,371-0845, Japan \\ ${ }^{5}$ Research Institute of Science and Technology, Tokai University, 2-28 Tomigaya, Shibuya-ku,Tokyo, \\ 151,Japan \\ ${ }^{*}$ Railway Technical Reseanch Institute, 2-8-38 hikari-a-cho,Kokubunji-City,Tokyo, 185,Japan
}

\begin{abstract}
It is known that a carbon fiber has a high biocompatibility. The amount of adhered activated sludge was much large when carbon fiber was bathed into an activated sludge. Activity and wet ability on surface of carbon fiber were increased by photo-oxygenation treated at $100^{\circ} \mathrm{C}, 150^{\circ} \mathrm{C}$, and $200^{\circ} \mathrm{C}$. After the treatment, functional groups including atoms were introduced to surface of carbon fiber. The amount of adhered activated sludge to treated carbon fiber was measured each at 1 minute. The amount of adhered activated sludge was increased with increasing treated temperature. The surface of carbon fiber was analyzed by X-ray photo-electronic spectroscopy (XPS). The ratio of oxygen/carbon atoms on the surface of treated carbon fiber was larger than that of untreated carbon fiber. Functional groups including oxygen introduced into the carbon fiber surface were influenced to the adhesion of activated sludge on carbon fibers and improved in the wet ability.
\end{abstract}

Keywords: Photo-oxygenation, Carbon Fiber, Activated Sludge, Biocompatibility

\section{Introduction}

Carbon fiber is used as reinforced materials of composite material of CFRP and CFRC. Then, adhesion of surface between carbon fiber and matrix give a great influence to mechanical strength. Therefore, for carbon fiber as goods on the market used, the surface treatment that we respond in a purpose is done. As we do surface treated to a carbon fiber, the shearing stress of composite material of carbon fiber is advanced. Generally, an modulus of elasticity of carbon fiber indicates a large shearing stress of composite material which gets weak, Use of a composite material for solving a problem is profitable as a purpose to improve it.

Surface treated to carbon fiber shows roughly two types. One is an introduction of functional groups including oxygen and etching on oxidation, and another is a method to attach materials to surface of carbonic fiber by graft or various coating.

Functional groups that occurs in case of the former, though it differs from a condition, are carboxyl group, phenol group, carbonyl group, ether bond and nitroso group [1]. For the above purposes, there are a reagent liquid oxidation method, electrolysis method[2] and gas phase oxidation. Reagent liquid oxidation methods and electrolysis method have contamination defects of samples and washing processing for post treatment, because this reaction is proceeded in liquid phase. On the other hand, gas phase oxidation is carried out under a condition, oxidation $16 \sim 30$ hours in air at $400^{\circ} \mathrm{C}$ [3] , and a surface modify method uses 
low temperature plasma [4]. However, this method is practicable only to a portion to need a hightemperature furnace and vacuum equipment, and the reaction is difficult to control [5] . In various gas phase oxidation methods, we select a photooxygenation method.

This method manages an oxidation raising a reaction activity of ozone further by ultraviolet rays irradiation, and raising ozone concentration. This method has a merit to be able to modify surface well with low temperature efficiency. In order to raise ozone concentration, ozone is generated from pure oxygen. We occurred photolysis responded by ultraviolet rays irradiation to raise a reaction activity of ozone and take atoms formed oxygen ( $\mathrm{O}$ ) and $\left(^{1} \mathrm{O}_{2}\right)$. Surface of carbon fiber is modified with ozone, $\mathrm{O}$, and $\left({ }^{\mathrm{l}} \mathrm{O}_{2}\right)$ which give very strong oxidation. In addition, this method is applied to photo-resist removing technology in semiconductor industries, being able to remove organic materials with an expensive efficiency for electric without damaging it [6]. Furthermore, in case of carbon fiber surface modified by photooxygenation, it is well known that carbon fiber is getting wet, and therefore continuous processing of photo-oxygenation method[7] is investigated. Yoshikawa et al [8] are tried to make clear dependencies of the carbon fiber surface modified by photo-oxygenation. Then, surface of carbon fiber introduced functional groups including oxygen improves a wet ability against water and a mechanical strength of a carbon fiber / concrete composite material is increased.

It was reported that CFRP is formed from the matrix to use phenol resin and epoxy resin, and the reinforced materials of the carbon fiber by photooxygenation increase flexural rigidity and shearing stress [9]. It was also reported that a strength of layered board is increased, when surface of a lumber is modified by photo-oxygenation in short time [10].

As seen above, by controlling an adhesive situation of a surface with a reinforced materials and matrix, a strength of a composite material is improved. On the other hand, when a carbon fiber bundle of strand was bathed inside the activated sludge, it is observed that an activated sludge adhesion tightly in large quantities with a short time. As we also make bathe pond and river which contaminate a carbon fiber bundle, an adhesion formed material was tightly wearable for the surface [11]. Then, contamination of sea is improved in remarkably degree in water $[12,13]$. However why they are adhesive to carbon fiber is not known. In order to purify quality of water quickly and more efficiency, we thought strongly that a bio-film superficially with respect to a carbon fiber is formed in large quantities. Therefore, it is necessary to improve wet ability between carbon fiber and micro-organism, in other words between carbon fiber and water. We also improve adhesive and wet abilities between carbon fiber and micro-organism by modifying surface of the carbon fiber by photo-oxygenation. Concretely, we used a photo-oxygenation.

Since an inclusion of oxygen functional group is well induced, we can revise and inquire hydrophilic group for a surface of a carbon fiber with a dryness style method. Then, a purpose of this study is that a surface modify condition ( treated temperature, and treated time ) of a carbon fiber is tightly wearable of a microorganism. In the present report, what kind of influence we give to quantity (tightly wearable of an activated sludge quantity ) is investigated.

\section{Method}

2.1.Surface modification.

For surface modification of a carbon fiber, UV dry stripper ( SAMUKO International Research Laboratory, UV-1 ) is used (Fig. 1). Oxygen is used for generation of ozone. Ultraviolet rays was irradiated through a mercury lamp. The wavelength used was $253.7 \mathrm{~nm}$ (about $90 \%$ ) and $184.9 \mathrm{~nm}$ (about 10\%). The substrate put on the fiber of the device is a disk made of aluminum of $200 \mathrm{~mm}$, internally a heater cooperated with it in crowds. The disk is able to heat materials from a room temperature up to 300 ${ }^{\circ} \mathrm{C}$. Treated temperature is $100{ }^{\circ} \mathrm{C}, 150{ }^{\circ} \mathrm{C}$ and

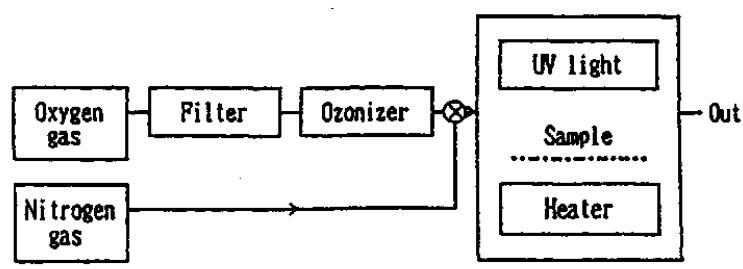

Fig.1 Schematic illustration of photo-oxygenation treatment 
treated time was 30 minutes or 60 minutes. As a surface of carbon fiber was partial irradiated to ultraviolet rays, arrangement of positions of a carbon fiber strand was changed in every 15 minutes. A remaining ozone after treatment was purged with nitrogen gas.

\subsection{Sample.}

A carbon fiber used is a strand type ( PAN type TOURE T-300,12K ). A carbon fiber was cut by a length $30 \mathrm{~cm}$. We fixed the upper part and the central part of the fiber with a tape. As surface of a carbon fiber strand was sized, it was bathed for two minutes in acetone and removed from sizing.

\subsection{Measurement of quantity of adherence to} activated sludge.

A quantity of adherence of a micro-organism to a carbon fiber was evaluated by a quantity of adherence of an activated sludge.

Protozoa, fungi and ameba such as small living things are useful for micro-organism groups in activated sludge for waste water treatment use. Consequently, we thought that with increasing a quantity of adherence of an activated sludge to carbon fiber, a quantity of adherence of a these

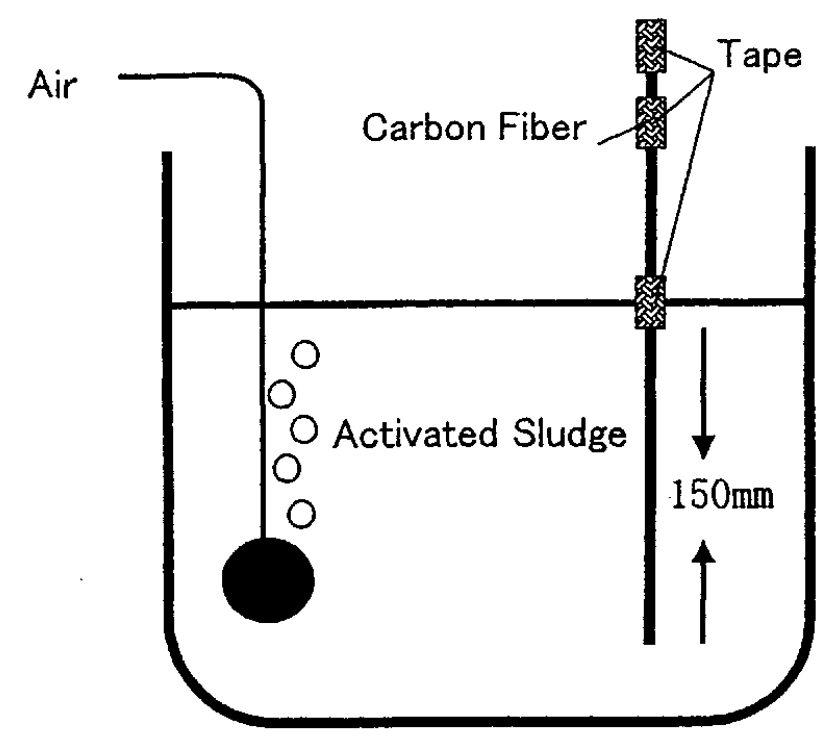

Fig.2. Carbon fiber bathed in the activated sludge. micro-organism is increased. We observed by an optical microscope that a portion of a microorganism group was included inside the activated sludge. A summary of a measurement method is shown in Fig. 2.

An activated sludge was included in 5-liter wide mouth bottle with air blow, about $1 \mathrm{liter} / \mathrm{min}$ ( that is, the activated sludge was diluted with water for 1.7 times using food bird factory use ). Inside the activated sludge, we made a lower part 15 $\mathrm{cm}$ of the carbon fiber strand to revise and inquire a surface bathed into it.

A carbon fiber strand was heaved for 1 minute after removing activated sludge, and measured the mass after falling drops. The activated sludge was bathed inside, then, heaved it for 1 minute, and the carbon fiber strand was weighed. Until 10 minutes the carbon fiber strand was weighed at each minute. For the purpose of a comparison, untreated carbon fiber strand was weighed. It was measured with a state that was not stripped, even though a sample was heaved from an activated sludge. It is requested that a quantity of adherence is estimated by subtracting a mass of the state that a carbon fiber gets wet from a mass of a carbon fiber.

\subsection{Analysis of surface of carbon fiber with XPS.}

Surface of carbon fiber modified by photooxygenation was measured with ESCA ( KRATOS AXIS-HS Shimazu ). Treated temperature was 100 ${ }^{\circ} \mathrm{C}, 150{ }^{\circ} \mathrm{C}$, and $200{ }^{\circ} \mathrm{C}$ for a surface condition of the modified carbon fiber. Treated time was 30 minutes. Untreated carbon fiber was used for the purpose of a comparison. The carbon fiber was washed for two minutes in acetone and removed from sizing. We measured oxygen and carbon ratio existence ( $\mathrm{O} / \mathrm{C}$ value ) at surface of carbon fiber. An inclusive oxygen functional group quantity was measured by separating wave of $1 \mathrm{~S}$ peak.

\section{Result}

3.1. Improvement of wet ability by surface modification.

An outside appearance of the carbon fiber by photo-oxygenation was not especially changed. However, in case of bathing carbon fiber in the water, a carbon fiber by photo-oxygenation was able easily to get wet, and settling at once. At any 
treated temperature and time, settling speed of carbon fiber in the water was not different.

\subsection{Quantity of adherence to activated sludge.}

Carbon fiber strand was bathed in activated sludge. As air blowing, every carbon fiber filament was dispersed and expanded in an activated sludge. Loosely a mass of a sample is increased as time passing. Comparing it before bathing, diameter of sample got fat and the whole got expanded. An increase was found in mass of the carbon fiber by photo-oxygenation at $100{ }^{\circ} \mathrm{C}$

Though mass of carbon fiber by photooxygenation for 60 minute and 30 minute was increased, a remarkable difference could not observed comparing with the untreated.

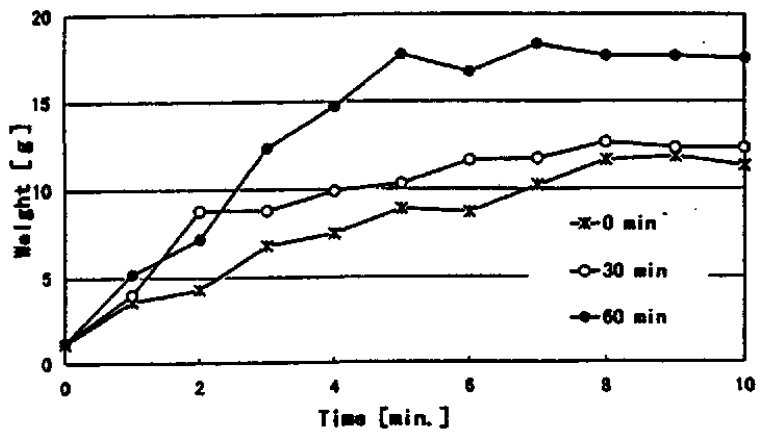

Fig.3. Weight change of carbon fiber bathed in the activated sludge by photo-oxygenation at $150^{\circ} \mathrm{C}$.

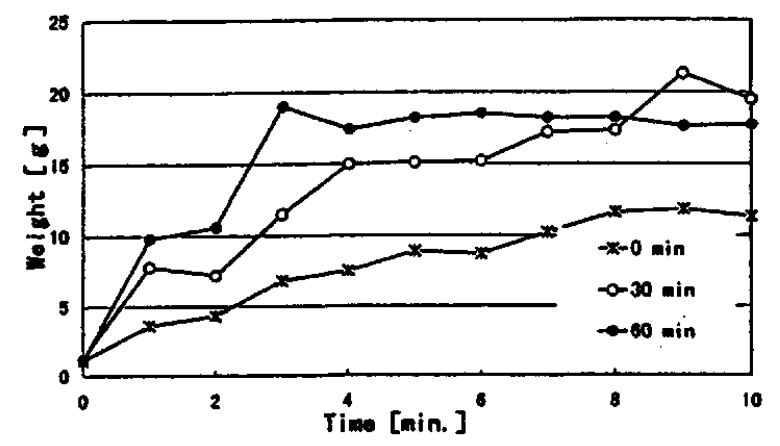

Fig.4. Weight change of carbon fiber bathed in the activated sludge by photo-oxygenation at $200^{\circ} \mathrm{C}$.
A mass increase of the carbon fiber by photooxygenation at $150{ }^{\circ} \mathrm{C}$ is shown in Fig. 3, where treated and untreated samples are compared: mass of treated carbon fiber, $18 \mathrm{~g}$, was 1.5 time than that of untreated carbon, $12 \mathrm{~g}$. A mass increase of the carbon fiber by photo-oxygenation at $200{ }^{\circ} \mathrm{C}$ is shown in Fig.4. The sample treated at $200{ }^{\circ} \mathrm{C}$ showed a tendency similarly to the sample treated at $150{ }^{\circ} \mathrm{C}$. For the carbon fiber treated at a higher temperature by photo-oxygenation, a quantity of adherence of an activated sludge was increased. In this case, a mass increase of a carbon fiber was independent from a quantitative of water, whether absorbs or wears it. With increasing in mass of a carbon fiber, quantity of absorption in water was increased.

For carbon fiber by photo-oxygenation bathed in water, mass of carbon fiber is related with time. In case bathed in water for ten minutes, an increase in mass of treated carbon fiber for $\mathbf{3 0}$ minute at $150{ }^{\circ} \mathrm{C}$ was not observed. In the same way, an increase in mass of untreated carbon was not either observed. They are not different from quantities by absorbing.

\subsection{Analysis of surface of carbon fiber by XPS.}

In a surface of carbon fiber by photooxygenation, kind and quantity of atoms were analyzed by XPS. $C_{1 S}$ spectrum is shown in Fig. 5 in separating wave. Typical spectra are seen in this figure, ether type oxygen (C-O), carbonyl type oxygen $(\mathrm{C}=\mathrm{O})$, and carboxyl type oxygen $\left(\mathrm{CO}_{2}\right)$ irrespective of the treated or the untreated sample. Their quantity did not depend on a difference of a treated temperature. $\mathrm{O} / \mathrm{C}$ value and oxygen functional group quantity are shown in Table 1. $\mathrm{O} / \mathrm{C}$ value of treated sample was increased approximately in 0.3 times, while that of untreated sample in 0.26 . When the treated temperature was higher, $\mathrm{O} / \mathrm{C}$ value was lower. A difference between the treated and the untreated was found in amount of C-C bond and quantity of inclusive oxygen functional group. C-C bond and carboxyl functional group was increased by photooxygenation, and ethereal bond was slightly increased. 


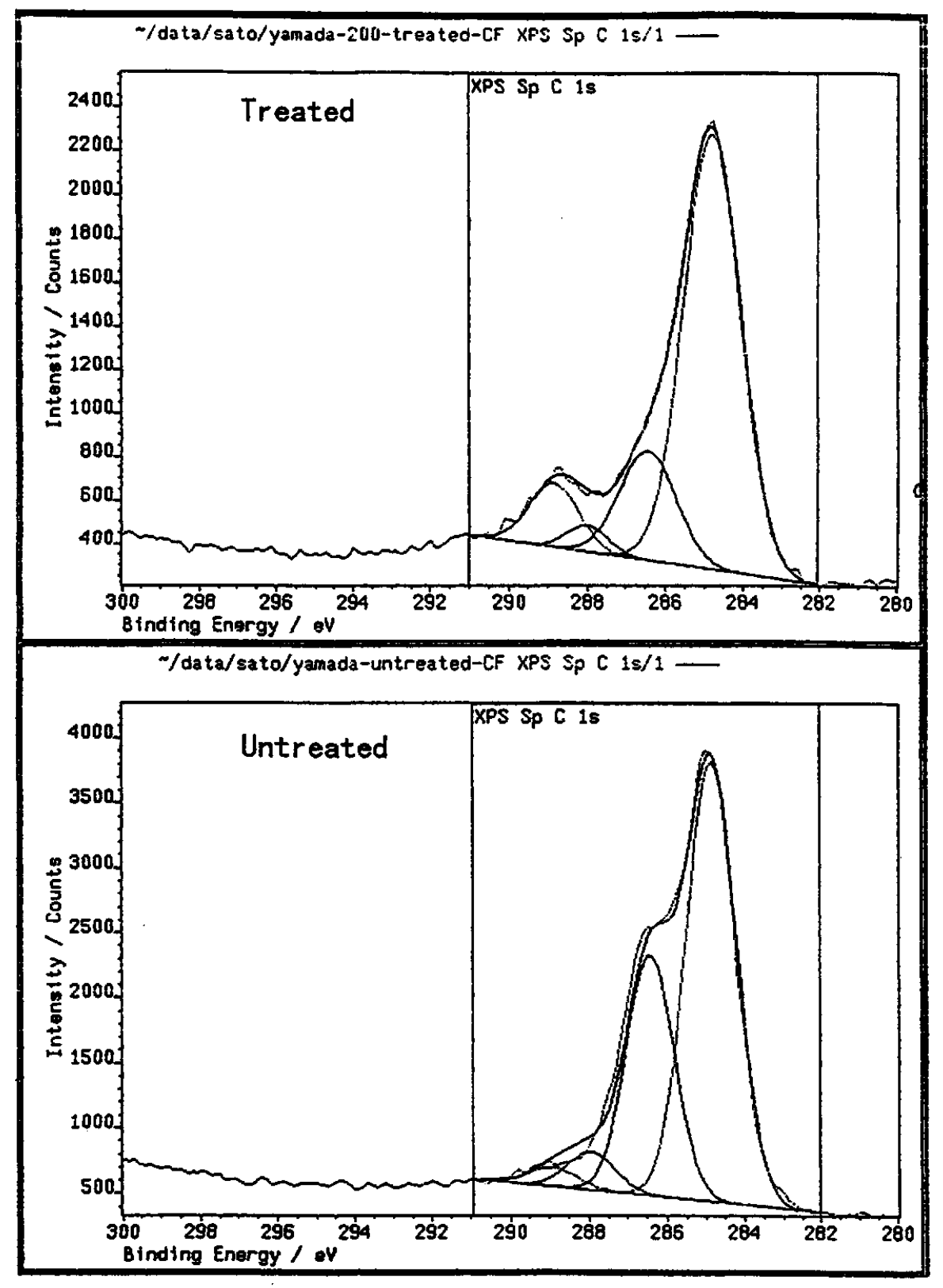

Fig.5. $C_{\text {is }}$ spectra of treated carbon fiber and untreated carbon fiber.

Table.1. Surface analysis of carbon fiber by XPS.

\begin{tabular}{|c|c|c|c|c|c|c|}
\hline \multirow[t]{2}{*}{ Treatment } & & \multirow[t]{2}{*}{$\mathbf{O} / \mathrm{C}$} & \multicolumn{4}{|c|}{ Functional group(\%) } \\
\hline & & & $C-C$ & $c-0$ & $C=0$ & $\mathrm{CO2}$ \\
\hline untreated & & 0.26 & 61.1 & 31.5 & 4.9 & 2.5 \\
\hline photo- & $100^{\circ} \mathrm{C}$ & 0.35 & 56.6 & 24.6 & 9.6 & 9,3 \\
\hline oxygenate & $150^{\circ} \mathrm{C}$ & 0.31 & 66.5 & 16.9 & 5.3 & 11.3 \\
\hline (30min.) & $200^{\circ} \mathrm{C}$ & 0.30 & 68.8 & 18.3 & 2.8 & 9.1 \\
\hline
\end{tabular}




\section{Discussion}

An outside appearance of PAN carbon fiber by photo-oxygenation was not especially changed in comparison with the untreated. In case of treatment at $150^{\circ} \mathrm{C}$, it is observed by SEM that a surface of a carbon fiber is not remarkably changed. However, in case of treatment at $250^{\circ} \mathrm{C}$, it is observed that surface of a carbon fiber was etched [14]. We treated it at temperature from $100{ }^{\circ} \mathrm{C}$ to $200{ }^{\circ} \mathrm{C}$. Therefore, it is estimated that surface of a carbon fiber is not irrespective of the treated or the untreated. Quantity of absorption was not remarkably different and irrespective of the treated or the untreated. Consequently, an increase in mass of a carbon fiber is caused by an activated sludge including a micro-organism. By analysis of surface of carbon fiber with XPS, $C_{1 S}$ spectra of the treated and untreated samples were observed and $\mathrm{O} / \mathrm{C}$ values were obtained. $\mathrm{O} / \mathrm{C}$ value of the untreated carbon fiber surface was 0.26 , while $\mathrm{O} / \mathrm{C}$ value of the treated at $100^{\circ} \mathrm{C}$ was 0.35 . On the other hand, $\mathrm{O} / \mathrm{C}$ value was 0.31 at $150^{\circ} \mathrm{C}$, while 0.30 at $200{ }^{\circ} \mathrm{C}$. It is shown that an atomic quantity of oxygen at treated carbon fiber surface is increased at $100^{\circ} \mathrm{C}$, and decreased with a temperature above $100{ }^{\circ} \mathrm{C}$. $\mathrm{C}-\mathrm{C}$ bond of the treated carbon fiber surface was increased, while $\mathrm{C}-\mathrm{O}$ bond (ethereal bond) decreased. A sizing was generally applied to a carbon fiber by epoxy resin. Consequently, the decrease of ethereal bond is caused by removing from sizing.

It is estimated that the untreated carbon fiber and the carbon fiber treated by photo-oxygenation at $100^{\circ} \mathrm{C}$ does not remove sizing superficially. For carbon fiber by photo-oxygenation at $150^{\circ} \mathrm{C}$ or $200{ }^{\circ} \mathrm{C}$, superficial oxidation was performed and sizing was removed off. At the same time, carboxyl group and carbonyl group were newly formed. When oxidation speed was greatly influenced by a treated temperature, for the carbon fiber by photo-oxygenation at $150^{\circ} \mathrm{C}$ or $200^{\circ} \mathrm{C}$, sizing was removed, e.g. quantity of carboxyl group $\left(\mathrm{CO}_{2}\right)$ was increased about $10 \%$ (treated at $200^{\circ} \mathrm{C}$ ) from $2 \%$ (untreated). When surface of carbon fiber was introduced by inclusive oxygen functional group, wet ability was advanced.
When advanced biocompatibility was performed, quantity of adherence to activated sludge was increased. In a case of $200^{\circ} \mathrm{C}$ and 150 ${ }^{\circ} \mathrm{C}$, while treatment time was longer, adhesion to activated sludge in the initial stage was more rapid, and quantity of adhesion was increased. On surface of carbon fiber quantity of functional group was increased, because of an advanced oxidation reaction. Furthermore, when an amino functional group is introduced, a different adhered bacteria phase is recognized. In near future, introduction of an inclusive nitrogen atomic group must be necessary.

Advances in wet ability, superficial electric potential and carbonic atom peculiar $\pi$ electron are important for carbon fibers. They were factors of adhesion to activated sludge in the initial rapid stage and quantity of adhesion was increased. Regarding these problems, the above step is a future theme. A correlative relation with a quality of water purification ability and microorganism quantity of adherence is another future theme.

\section{References}

1. S.Otani et al., "CARBON FIBRE", 217.

2. P.Ehrburger et al., "Tansobunken-Center, 76-78.

3. J.W.Johnson, SAMPE Symp., 12 (1967), AC-8.

4. A.Kojima et al., TANSO, 14 (1989), 261-267.

5. T.Yoshikawa, RTRI REPORT, 3 (1995), 2.

6. O.Tsuji, Semiconductor International, 11(1988), 58-9.

7. T.Yoshikawa, RTRI REPORT, 3 (1995), 164-6.

8. T.Yoshikawa et al., MATERIAL, 462 (1992), 369-74.

9. A.Kojima et al., TANSO, 162 (1994), 71-7.

10. K.Shimizu et al., J. Adhesion, 29 (1993), 268-72.

11. A.Kojima et al., Gakujutsusinkoukai, 117233-C1(1995)

12. A.Kojima et al., Gakujutsusinkoukai, 117-235B1(1995)

13. A.Kojima et al., Carbon Material Summer Seminar 34 (1996), 88.

14. T.Yoshikawa, RTRI REPORT, 3 (1995), 22. 